\title{
Topology and weights in a protein domain interaction network - a novel way to predict protein interactions Stefan Wuchty*
}

\author{
Address: Northwestern Institute on Complexity, Northwestern University, 600 Foster Street, Evanston, IL 60208, USA \\ Email: Stefan Wuchty* - s-wuchty@northwestern.edu \\ * Corresponding author
}

Published: 23 May 2006

BMC Genomics 2006, 7:122 doi:10.1186/147|-2164-7-122
Received: 3I January 2006

Accepted: 23 May 2006

This article is available from: http://www.biomedcentral.com/I47I-2/64/7//22

(c) 2006 Wuchty; licensee BioMed Central Ltd.

This is an Open Access article distributed under the terms of the Creative Commons Attribution License (http://creativecommons.org/licenses/by/2.0), which permits unrestricted use, distribution, and reproduction in any medium, provided the original work is properly cited.

\begin{abstract}
Background: While the analysis of unweighted biological webs as diverse as genetic, protein and metabolic networks allowed spectacular insights in the inner workings of a cell, biological networks are not only determined by their static grid of links. In fact, we expect that the heterogeneity in the utilization of connections has a major impact on the organization of cellular activities as well.

Results: We consider a web of interactions between protein domains of the Protein Family database (PFAM), which are weighted by a probability score. We apply metrics that combine the static layout and the weights of the underlying interactions. We observe that unweighted measures as well as their weighted counterparts largely share the same trends in the underlying domain interaction network. However, we only find weak signals that weights and the static grid of interactions are connected entities. Therefore assuming that a protein interaction is governed by a single domain interaction, we observe strong and significant correlations of the highest scoring domain interaction and the confidence of protein interactions in the underlying interactions of yeast and fly.

Modeling an interaction between proteins if we find a high scoring protein domain interaction we obtain I, 428 protein interactions among $36 \mathrm{I}$ proteins in the human malaria parasite Plasmodium falciparum. Assessing their quality by a logistic regression method we observe that increasing confidence of predicted interactions is accompanied by high scoring domain interactions and elevated levels of functional similarity and evolutionary conservation.

Conclusion: Our results indicate that probability scores are randomly distributed, allowing to treat static grid and weights of domain interactions as separate entities. In particular, these finding confirms earlier observations that a protein interaction is a matter of a single interaction event on domain level. As an immediate application, we show a simple way to predict potential protein interactions by utilizing expectation scores of single domain interactions.
\end{abstract}

\section{Background}

The depiction of interactions between genes, proteins and metabolites as networks has uncovered unexpected similarities in the organization of various biological networks, indicating that generic principles and mechanics give rise to their structure. Although such networks vary extensively in their complexity, corroborative evidence points to a series of simple organizing principles that character- 
ize all complex networks. The most dramatic is the scalefree nature of these networks, a remarkable inhomogeneity that highlights a small number of highly connected nodes which secure the networks integrity [1]. The special role such proteins play for the stability of protein interaction networks is further indicated by their significant propensity to be simultaneously essential as well as evolutionary conserved [2]. Reflecting their inherent cohesive nature, complex networks are characterized by the accumulation of discernible modules. Such clusters of densely interconnected nodes combine in an overlapping manner, share well defined functions and hubs as the modules connectors $[1,3,4]$. Similarly to hubs, cohesively bound motifs of protein networks are frequently conserved as a whole, suggesting their role as evolutionary relevant units [5]. While these findings allowed spectacular insights into the inner workings of a cell, biological networks are generally not only determined by their layout of links. In fact, we expect that the heterogeneity in the utilization of connections has a major impact on the organization of cellular activities as well. Recently, attention turned to weighted scientific collaborations and airways networks [6], allowing a first insight into the intricate interplay between links and their weights. Concluding, analysis of real world networks indicate that the static grid of links and their weights can not be regarded as separate entities. Here, we present a first statistical analysis of a weighted biological network by considering a web of PFAM domain interactions. Each link between domains is weighted by an expectation score, reflecting the probability that a particular domain interaction indeed gives rise to observed protein interactions. Applying metrics that combine the static layout of interactions and their weights, we observe that the patterns of correlations are similar for weighted and unweighted network parameters. In contrast to other real world networks, we find weak signals that do not support an entanglement of static grid and weights of domain interactions, allowing us to confirm that a protein interactions are largely governed by single domain interactions.

Assuming that pairs of interacting proteins in S. cerevisiae and $D$. melanogaster are indeed dominated by the highest scoring domain interaction their domain architectures suggest, we find that the confidence score of a protein interaction correlates well with its highest scoring domain interaction. As an application, this observation indicates a simple method to model interactions between proteins of the human malaria parasite P. falciparum. Assuming an interaction between proteins if we find at least one high scoring domain interaction we predict 1,428 novel protein interactions among 321 proteins. The quality of each predicted interaction is assessed by a logistic regression model, allowing us to uncover reliable interactions between proteins that share similar functions and are preferably conserved in evolution.

\section{Results}

As a source of high quality interaction data of protein domains we utilized the results of a recent study by Riley et al. [7]. In this statistical approach, called domain pair exclusion analysis (DPEA), a likelihood ratio test is applied to assess the contribution of each potential PFAMA and PFAM-B domain [8] interaction to the likelihood of a set of observed protein interactions as of DIP [9]. Applying a statistical framework which evaluates the confidence that domains $i$ and $j$ indeed interact, the authors obtain a network of 1, 566 domains that are embedded in a web of 2, 767 interactions. Weighting each interaction by its probability score - the expectation value [7] - we are primarily interested in the interplay between topology and the reliability of the underlying interactions.

Allowing a first insight in the weights role, we observe a heavy tail in the cumulative distribution of the expectation value of domain links $E$, which can be roughly approximated by a power-law $\left(P(E) \sim E^{-2.7}\right)$ (Figure 1a). In real world networks the correlation of the degree product $k_{i} k_{j}$ and the weight $w_{i j}$ follows a power-law shaped curve, potentially indicating an intricate relationship between the static layout and weights of links. In our case, we hardly find such a dependence (Figure 1a, inset). In fact, the mean expectation value is almost constant for more than two decades, indicating a general lack of correlation between weights and the domains number of interaction partners [6]

Investigating further if the topology of the underlying domain interaction network and their weights are indeed independent from each other, we combine both topology and weights by a series of measures that enable a more significant assessment of the impact of weights [6]. In an unweighted domain interaction network, the domains degree is defined as $k_{i}=\sum_{j} a_{i j}$ where $a_{i j}=1$ if there exists a link between domains $i$ and $j$. Extending this definition, the strength of a domain $i$ is defined as

$s_{i}=\sum_{j} a_{i j} E_{i j}$

accounting for individual expectation values $E_{i j}$ as weights of interactions of domain $i$. Comparing the statistical properties of a domains degree $k$ and its strength $s$ we observe that their frequency distributions follow a generalized Zipf's law $P(x)=\alpha \times(\beta+x)^{-\gamma}$ (Figure 1b) [10]. The power-law tail of the degree distribution indicates the presence of scale-free topology [11], suggesting that the integrity of the underlying network basically depends on 

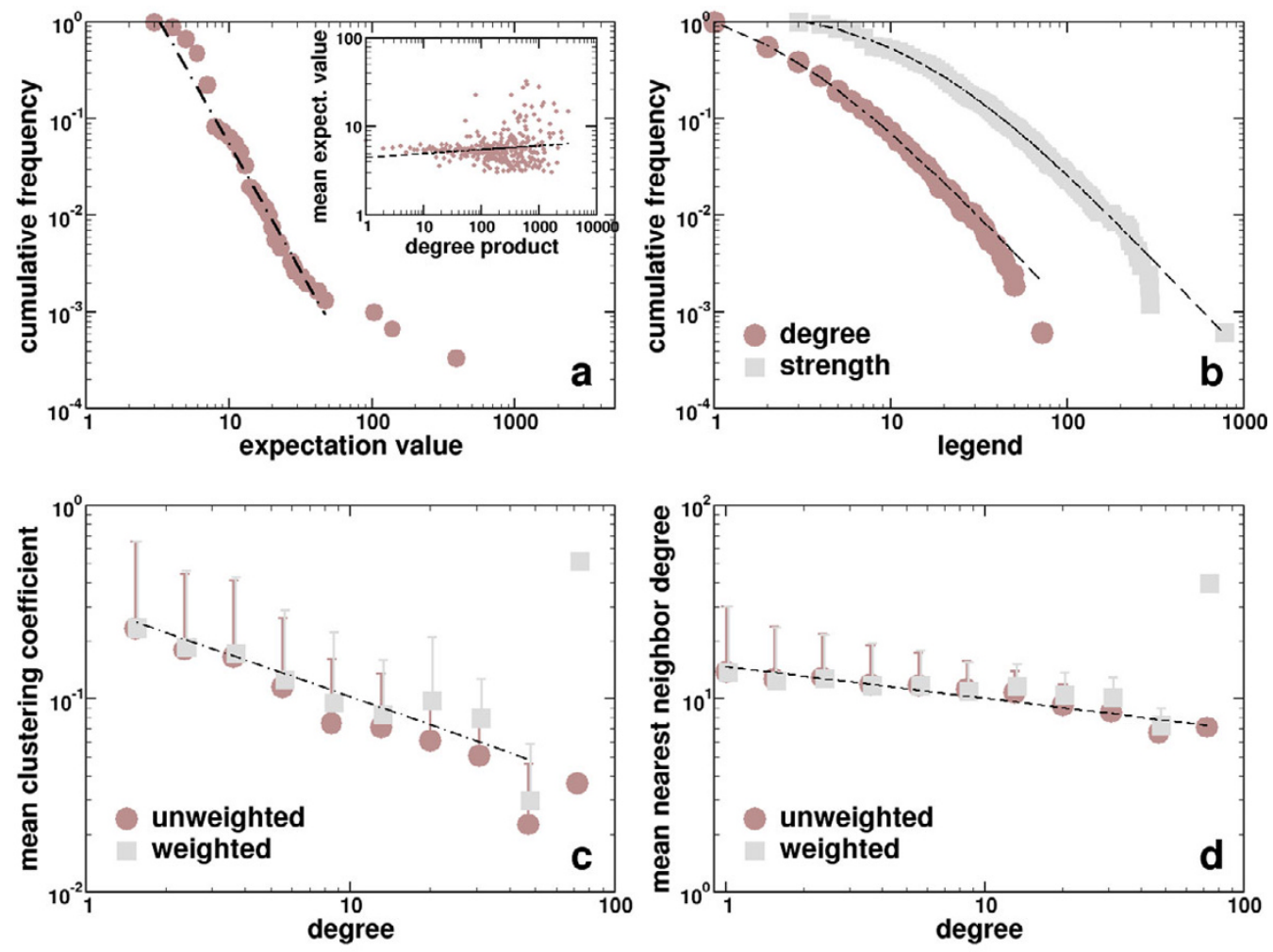

\section{Figure I}

Statistics of the domain interaction network. (a). In the cumulative distribution of the expectation value of domain interactions we observe a heavy tail. Focusing on lower ranges of the expectation value, we approximated a power-law $(P(E) \sim$ $\left.E^{-2.7}\right)$. The dependence of an interactions expectation value from the product of the domains degree $k_{i} k_{j}$ shows a weak correla-

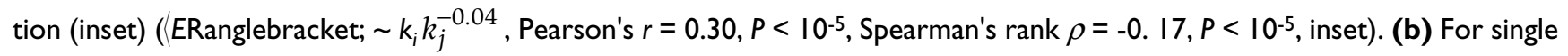
domain based measures such as the degree $k$ and the strength $s$, we observe power-law tailed cumulative frequency distributions as well. Both distributions follow a generalized Zipf's law $\left(P(k)=8.7 \times(2.1+k)^{-1.9}, P(s)=298.0 \times(I 4.6+s)^{-2.0}\right)$. (c) Indicating a networks modularity, the dependence of the clustering coeffcient $C$ decays as a power-law, $C(k) \sim k^{-0.55}$. Basically, we observe the same correlation for the weighted clustering coeffcient $C^{w}(k) \sim k^{-0.47}$, indicating that the weighted generalization of the clustering coeffcient does not change the initial correlations. (d) The unweighted average nearest neighbor degree slightly decays with increasing degree. This albeit weak dependency is roughly approximated by a power-law $\left(k_{n n} \sim k^{-0.16}\right)$. In principle, we obtain the same result for the weighted representation as well $\left(k_{n n}^{w} \sim k^{-0.1}\right)$. In (c) and (d), we logarithmically binned the data points and calculated mean values and standard deviations in each bin.

a small subset of highly connected nodes. Analogously, there exists a majority of nodes having low strength while a minority of nodes reach high levels of strength. A list of highest interacting domains shows prominent protagonists that are responsible for important cellular functions such as signaling and cell-cell contacts (Table 1 ). In particular, we observe that highly connected domains such as pkinase, rrm1 or Zinc finger $\mathrm{C} 2 \mathrm{H} 2$ also pool a lot of strength, indicating a proportionality between high level of interactions and their strength.
Investigating the local cohesiveness of network areas, the unweighted representation of the clustering coeffcient $C_{i}$ measures the degree of cohesiveness around a particular domain $i$ [12]. The dependence of the average clustering coeffcient $C$ from the domains degree $k$ recovers further information about the structure of the underlying network. In most real world networks $C(k)$ exhibits a highly nontrivial behavior as exemplified by a power-law decay with increasing degree $k$. Averaging over the clustering coeffcients of domains with a certain degree $k$, we find this particular signature, indicating the presence of a nested 
Table I: Statistics of single domains. Domains in the underlying interaction network are characterized according to their degree $k$ and their strength $s$, defined as the sum of all weights the domain in question is involved in. Here, we show the 10 most connected and strongest PFAM domains.

\begin{tabular}{|c|c|c|c|c|c|}
\hline PFAM domain & description & degree $k$ & PFAM domain & description & strength $s$ \\
\hline PFOI423 & LSM & 72 & PFOI 423 & LSM & 777.7 \\
\hline PF0007I & ras & 50 & PF00II8 & TCP-I/cpn60 & 294.5 \\
\hline PF00022 & actin & 50 & PF00022 & actin & 291.5 \\
\hline PF00069 & pkinase & 49 & PF00069 & pkinase & 289.0 \\
\hline PF00076 & rrml & 45 & PF0007I & ras & 263.5 \\
\hline PF00II 8 & TCP-I/cpn60 & 43 & PF00076 & rrml & 253.4 \\
\hline PF00096 & $\mathrm{zf}-\mathrm{C} 2 \mathrm{H} 2$ & 39 & PB075870 & - & 248.8 \\
\hline PB075780 & - & 39 & PF00227 & proteasome & 237.1 \\
\hline PF00036 & efhand & 36 & PF0I008 & $\mathrm{IF}-2 \mathrm{~B}$ & 226.5 \\
\hline PF0I008 & IF-2B & 35 & PF0000I & $7 \mathrm{tm}-1$ & 226.0 \\
\hline
\end{tabular}

hierarchy of modules [1] (Figure 1c). Accounting for weights, Barrat et al. [6] extended the initial definition of the clustering coeffcient to combine topological information with weights of network links. Considering the expectation value of each domain interactions $E$ as the weight of links, we define the weighted clustering coeffcient as

$$
C_{i}^{w}=\frac{1}{s_{i}\left(k_{i}-1\right)} \sum_{j, h} \frac{E_{i j}+E_{i h}}{2} a_{i j} a_{i h} a_{j h} .
$$

Since the structure essentially follows the concept of the original clustering coeffcient, we expect that $C_{i}^{w}$ retains its dependence from the degree $k$. Indeed, we find a powerlaw dependence in both networks (Figure 1c). Considering the mean weighted clustering coeffcient of the whole network as the arithmetic mean over all domains $N$, $\left\langle C^{w}\right\rangle=\frac{1}{N} \sum_{i=1}^{N} C_{i}^{w}$, we obtain 0.097. Comparing this result to the value of the mean unweighted clustering coeffcient of 0.093, we find that 〈C ${ }^{w}$ Ranglebracket; $\langle$ CRanglebracket; $\approx 1.0$. Since the weighted clustering coeffcient reflects a domain's neighborhood to be connected to domains of similar strength the latter result indicates that local clustering predominately occurs on the level of comparable strength.

Another measure that allows insights in the relationship of network layout and weights are degree-degree correlations. Similarly to $C^{w}$, we define the average weighted nearest-neighbors degree as [6]

$k_{n n, i}^{w}=\frac{1}{s_{i}} \sum_{j} a_{i j} E_{i j} k_{j}$.
In an unweighted network the definition of $k_{n n, i}$ recovers the average nearest neighbor degree of a node, where $k_{n n, i}=\frac{1}{k_{i}} \sum_{j} a_{i j} k_{j}$. In the presence of correlations with connectivity $k$, the behavior of the latter measure $k_{n n, i}(k)$ identifies two classes of networks. If $k_{n n}(k)$ is an increasing function with $k$, vertices with higher degree have an increased probability to be connected with large-degree vertices, a feature that is known as assortative mixing. If $k_{n n}(k)$ decreases with $k$, the underlying network is disassortative, indicating that high degree vertices predominantly are connected to sparsely linked ones. Similarly to other biological networks [13], we find a weak albeit significant trend toward disassortativity in both the unweighted and weighted domain interaction networks (Figure 1d). Considering the nearest neighbor degree of the whole network as the arithmetic mean over all nodes $N,\left\langle k_{n n}^{w}\right\rangle=\frac{1}{N} \sum_{i=1}^{N} k_{n, i}^{w}$, we obtain 12.81. Comparing this result to the value of the mean unweighted nearest neighbor degree of 12.84, we find that 10216; $k_{n n}^{w} 10217$; $\kappa \_n n w \approx 1.0$, indeed confirming that in both the weighted as well as unweighted representation the disassortative behavior prevails.

The previously introduced topological measures of both unweighted and weighted representations of the same domain interaction network share the same qualitative features, suggesting that weights and topology are entangled entities. However, recalling the observation that the degree product does not correlate with the links underlying weights casts doubt on this assumption. Further insights into a potential interplay of topology and utilization of domain interactions arise from correlations 
between a domains degree and strength (Figure 2a). Despite the existence of inevitable fluctuations, the dependence of the strength from the degree of a domain in the underlying domain interaction network shows a clear and significant power-law $s(k) \sim k^{\beta}$ with $\beta=1.04$, allowing us to conclude that topology and utilization of links in domain interaction networks are separate entities since independent weights and connectivities would lead to an exponent $\beta=1$ [6]. We receive further support of this hypothesis by the disparity value $Y_{2}$, a measure that quantifies biased distributions, defined as

$$
Y_{2}(i)=\sum_{j \in \Gamma(i)} \frac{E_{i j}^{2}}{s_{i}^{2}}
$$

where $\Gamma(i)$ is the set of neighbors of domain $i$. In Figure $2 \mathrm{~b}$ we observe a clear power-law in the dependence of the disparity value $Y_{2}$ from the degree $k, Y_{2}(k) \sim k^{-0.9}$. Similarly to the dependence of the strength from the degree (Figure $2 \mathrm{a})$, an exponent close to 1 suggests that the expectation values of domain interactions are distributed in an uncorrelated manner $[6,14]$.

The absence of any correlations between the structure of the web of domain interactions and their confidence suggests that domain interactions hardly interfere with each other. As a consequence, protein interactions are primarily governed by a single domain interaction. Indeed, a recent survey of protein interactions uncovered a rate of
$94 \%$ that protein interactions are determined by a single pairwise domain interaction [15] while protein interactions that involve interactions between two or more domains are hardly found. A high $E$ reflects the probability that the domains in question indeed interact while a low $E_{i j}$ suggests that other potential domain interactions are roughly as good at explaining the observed protein interactions [7]. Therefore, we assume that a protein interaction is governed by the domain interaction with the highest expectation value. In order to uncover a potential correlation between the quality of a particular protein interaction and the highest scoring domain interaction, we utilize two well curated sets of protein interactions in $S$. cerevisiae [16] and D. melanogaster [17] where each interaction is evaluated by a confidence score. Utilizing information about the domain composition of proteins as of the Integr8 database, we screen each domain pair that is suggested by the domain architectures of the underlying proteins. Provided these pairs indeed map to high scoring domain interactions, each protein interaction is assumed to be governed by the domain interaction with highest expectation score. Applied to the evaluated protein interaction sets of S. cerevisae and D. melanogaster, we observe a strong and significant correlation between an interactions confidence and the expectation value of the underlying highest scoring domain interaction (Figure 4a). In turn, we can potentially use the previous conclusion that the absence of correlations between interactions and their probability indicates the dominance of single domain interactions as a means to infer protein interactions. As an
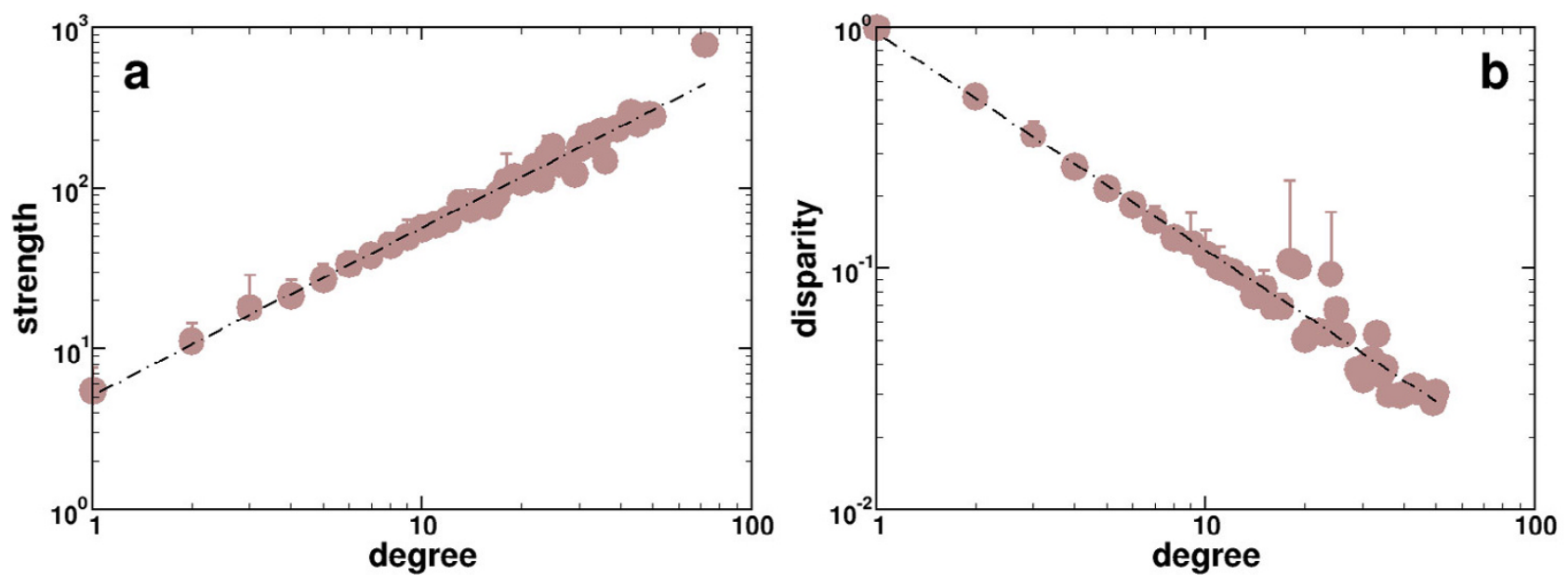

Figure 2

Statistics of domain strength. (a). The strength of a domain is defined as the sum of all expectation values of interactions a domain is involved in. The dependence of the strength from the domains degree shows a clear power-law $s(k) \sim k^{1.04}$ (Pearson's $r=0.94, P<10^{-5}$; Spearman's $\rho=0.93, P<10^{-5}$ ), suggesting that connectivity and weights are widely independent. (b) The disparity value offers further support for this conclusion, since we find that $Y_{2}(k) \sim k^{-0.9}$ (Pearson's $r=0.43, P<10^{-5}$; Spearman's $\left.\rho=0.88, P<10^{-5}\right)$. 


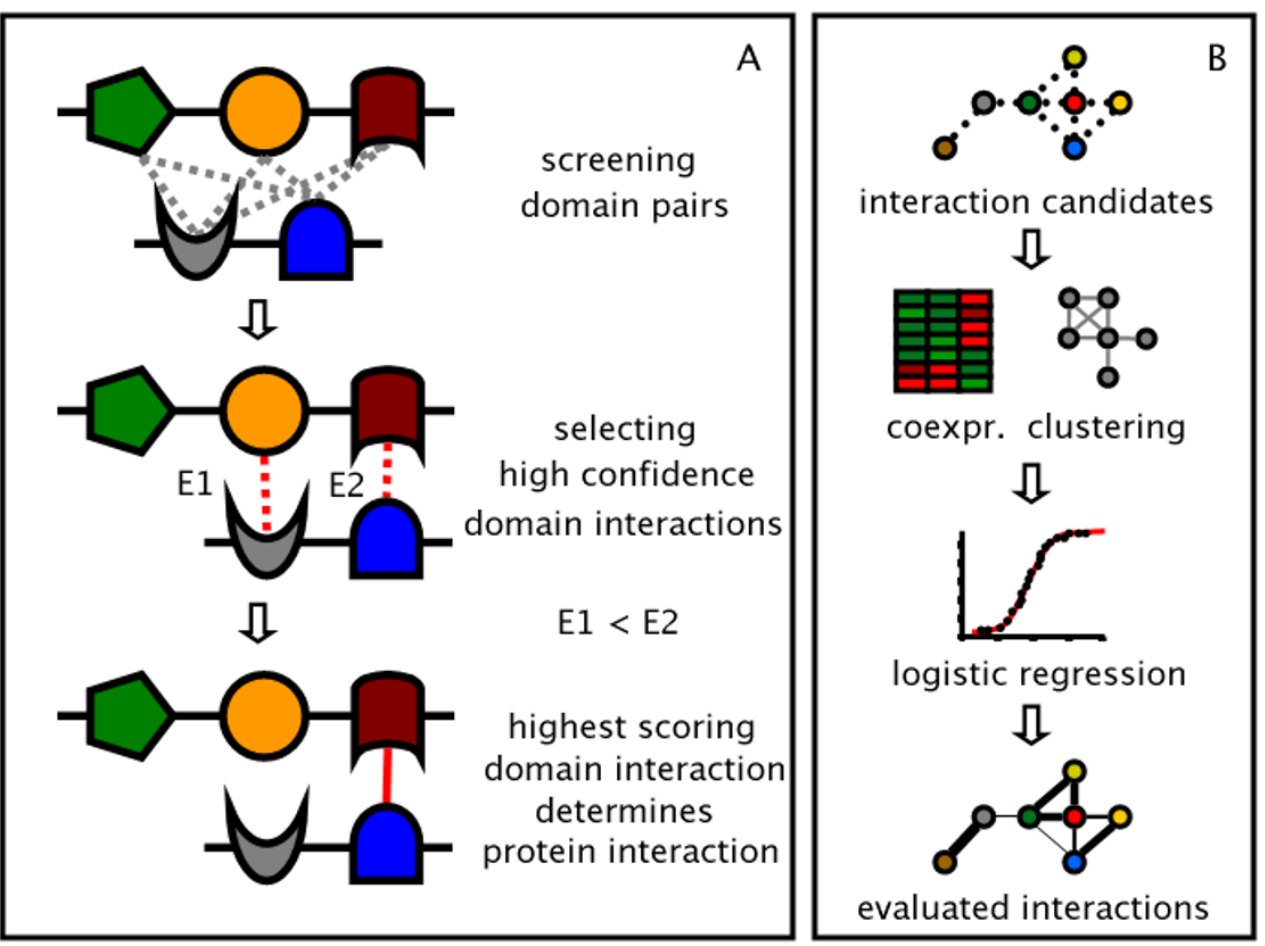

Figure 3

Schematic illustrations of prediction and evaluation procedures. (a). Each pair of proteins in $P$. falciparum that shares at least on GO Slim term of the cellular component annotation set is screened for all possible domain pairs. Comparing all interacting domain pairs according to their expectation value, we assume that the highest scoring domain interaction is governing the candidate interaction. (b) For each interaction candidate we calculate hypergeometric clustering coeffcients $C_{v w}$ and coexpression correlation coeffcients $r_{p}$. These parameters allow the domain independent assessment of a protein interaction by utilizing a logistic regression model.

organism, we chose the human malaria parasite $P$. falciparum. Utilizing domain information from the Integr8 database we annotate Plasmodium proteins with their corresponding PFAM domains. In order to avoid interactions between proteins that appear in different compartments we additionally assign each protein with its cellular component terms as of the GO Slim database [18]. Considering all protein pairs of Plasmodium we select those that share at least one GO Slim term. The domain architectures of candidate protein pairs are screened for domain pairs that have at least one high scoring domain interaction. In case we find more than one high scoring domain interaction, we choose the highest scoring one, according to the statistical argument that domain interactions with higher expectation score have a better chance to explain the underlying protein interaction. In Figure $3 \mathrm{a}$, we give a schematic survey of the procedure. Applying this method to the proteome of $P$. falciparum we find 1, 428 interactions between 361 proteins [see Additional file 1]. In order to evaluate each of these potential protein interactions, we characterize each link by measures that reflect biological significance. In particular, we are interested in parameters that are independent of the initial assumption that the highest scoring domain interaction indeed can be used to predict protein interactions. As such, we choose co-expression correlation values of interacting proteins, since similar expression profiles tend to indicate interacting proteins. For $P$. falciparum, we utilized gene expression data over 48 time points. Compiling gene expression data derived from micro-array analysis [19-21], we determine Pearson's correlation coeffcients $r_{P}$ of each interaction (see Materials and Methods). In addition, we calculated hypergeometric clustering coeffcients $C_{v w}$ for each interaction, a topological measure that reflects local cohesiveness around a certain link and strongly correlates with the quality of the underlying protein interaction [22] (see Material and Methods). Combining these measures, we utilized a logistic regression method (see Material and Methods) trained by carefully selected sets of 213 true positive and 173 negative interactions, allowing us to assess the quality of each interaction by a confidence score between 0 and 1 (Figure $3 \mathrm{~b}$ ). As a quality measure of the 
utilized training sets, we performed a leave-one-out strategy, allowing us to obtain 95\% accuracy.

Binning interactions according to their confidence value, we observe that about half of the interactions have an elevated degree of confidence (Figure $4 \mathrm{~b}$ ). In each bin, we averaged the expectation score of the domain interactions and observe that high quality of protein interactions - as exemplified by high confidence - are strongly linked to high expectation scores of the underlying domain interaction (Figure 5a). Supported by significant correlation values, this observation is a confirmation of our original assumption that protein interactions are dominated by the highest scoring domain interactions, while high scoring domain interactions indicate the presence of a potential protein interaction. As additional measures of quality, we make use of the well known fact that protein interactions occur between proteins of similar function [23]. As a measure of functional homogeneity of interacting proteins, we apply a hypergeometric test (see Materials and Methods) of the distributions of the proteins GO terms [18]. In particular, this statistical measure reflects the probability that GO terms of interacting proteins have been distributed randomly. Averaging over all interaction specific values in each bin, we find a strong and significant correlation, confirming that protein interactions of increasing confidence tend to occur between functionally related proteins (Figure 5b). As a final test, we wondered if the predicted protein interactions in P. falciparum have an evolutionary signature. In particular, we utilized three protein interaction sets of the organisms S. cerevisiae [16], D. melanogaster [17] and $H$. sapiens [23,24]. Utilizing orthologous protein information from the InParanoid database [25], we sampled all protein interactions in each organisms that have a fully conserved counterpart - an interolog [26] - in the predicted set of interactions of $P$. falciparum. In Figure 5c, we observe that especially predictions with high confidence pool most of the found interologs in each organism, strongly indicating the reliability of our predictions.

We compared the predicted sets of interactions to a recently published set of experimentally determined protein interactions of P. falciparum [27]. Although many interactions of this set have been assigned potential protein domain interactions, the utilized domain information does not overlap strongly with PFAM, restricting the overlap with our predicted set to only 2 interactions. In particular, we find self interactions of the hypothetical Plasmodium proteins PFL0275w and PF10_0232. In the first case, a self interaction of the FHA domain gives rise to the observed protein interaction, while a self interaction of chromo domain determines the latter one. In both cases, the interacting proteins are hypothetical, meaning that their function is unclear. However, the fact that we found domain interactions suggests a role for these proteins. In particular, the forkhead-associated FHA domain is a phosphopeptide recognition domain found in many regulatory proteins, while the chromo (CHRromatin Organization MOdifier) domain is a conserved region of around 60 amino acids involved in the alteration of the structure of chromatin. Putatively, PFL0275w is involved in regulatory activities while PF10_0232 might play a role in chromatin remodeling. In general, our predictions show a prevalence of functions revolving around the proteasome, spliceosome and ribosome. In particular, Table 2 ranks the domain interactions that gave rise to the highest number of predictions in P. falciparum. In particular, we observe that domain interactions between the RNA recognition motif rrm1, proteasome and LSM domains appear among the most prevalent domain interactions. As the previous examples illustrates, many interactions are related to self interactions of the underlying domains. As such, we observe a total of 154 self interactions. Indeed, it is well known that multi-protein complexes contain homo-dimers including proteasome [28], ribosome [29] and spliceosome [30]. In particular, rrm's are found in a variety of RNA binding proteins, including various hnRNP proteins, proteins implicated in regulation of alternative splicing, and protein components of snRNPs. The LSM domain contains Sm proteins as well as other related LSM (Like Sm) proteins. The U1, U2, U4/U6, and U5 small nuclear ribonucleoprotein particles (snRNPs) involved in pre-mRNA splicing contain seven Sm proteins in common, which assemble around the Sm site present in four of the major spliceosomal small nuclear RNAs. The U6 snRNP binds to the LSM (Like Sm) proteins. The proteasome is a multicatalytic proteinase complex that is involved in an ATP/ubiquitin-dependent proteolytic pathway. In eukaryotes, the proteasome is composed of about 28 distinct subunits, which form a highly ordered ring-shaped structure (20S ring). Concluding, in the proteasome, ribosome and spliceosome proteins which carry those domains tend to shape stable structures which are mostly governed by self domain interactions, validating the presence of self interactions in our predictions.

\section{Discussion \& conclusion}

Assessing the statistical characteristics of a weighted domain interaction network we show that the confidence as exemplified by the expectation value of domain interactions is far from being evenly distributed. Characterizing the underlying weighted domain interactions network, we observe that weighted and unweighted measures of topology follow the same trends. Despite these observations we do not find any significant proof that topology and weights in the domain interaction network are necessarily dependent from each other. In fact, correlations between strength and connectivity as well as disparity suggest that weights as exemplified by the 
Table 2: Domain interactions in predictions of protein interactions in Plasmodium. Predicting protein interactions by their highest scoring domain interaction in $P$. falciparum we find the following 20 most frequent domain interactions. $N$ refers to the domain interactions occurrence in the predicted set, $\%_{s l}$ depicts the percentage of self protein interactions, and $E$ is the expectation value of the underlying domain interaction.

\begin{tabular}{|c|c|c|c|c|c|c|}
\hline domain & description & domain & description & $N$ & $\%_{s l}$ & $E$ \\
\hline PF00076 & rrml & PF0I423 & LSM & 137 & - & 14.5 \\
\hline PF00227 & proteasome & PF00227 & proteasome & 120 & 12.5 & 103.1 \\
\hline PFOI423 & LSM & PFOI423 & LSM & 120 & 12.5 & 387.1 \\
\hline PF00005 & $A B C$ transporter & PF00005 & $A B C$ transporter & 83 & 16.5 & 4.9 \\
\hline PF00097 & $\mathrm{zf}-\mathrm{C} 3 \mathrm{HC} 4$ & PF00240 & ubiquitin & 74 & - & 5.7 \\
\hline PF00076 & rrm l & PF00076 & rrml & 56 & 28.7 & 14.5 \\
\hline PF00022 & actin & PF00022 & actin & 55 & 18.1 & 8.5 \\
\hline PF00I 25 & histone & PF00I 25 & histone & 36 & 22.1 & 11.6 \\
\hline PFOI423 & LSM & PF06220 & zf-UI & 30 & - & 20.3 \\
\hline PF02953 & $\begin{array}{l}\text { Tim I0/DDP zinc } \\
\text { finger }\end{array}$ & PF00I53 & $\begin{array}{l}\text { mitochondrial } \\
\text { carrier }\end{array}$ & 30 & - & 6.6 \\
\hline PF00097 & $\mathrm{zf}-\mathrm{C} 3 \mathrm{HC} 4$ & PFOI 283 & $\underset{S}{\text { Ribosomal protein }}$ & 24 & - & 3.0 \\
\hline PF00097 & $\mathrm{zf}-\mathrm{C} 3 \mathrm{HC} 4$ & PF0I775 & Ribosomal LI8ae & 24 & - & 7.3 \\
\hline PF00097 & $\mathrm{zf}-\mathrm{C} 3 \mathrm{HC} 4$ & PF00833 & Ribosomal SI7 & 24 & - & 3.6 \\
\hline PF00097 & $\mathrm{zf}-\mathrm{C} 3 \mathrm{HC} 4$ & PF00827 & Ribosomal LI5 & 24 & - & 3.2 \\
\hline PFO0II8 & $\begin{array}{l}\text { TCP-I/cpn60 } \\
\text { chaperonin }\end{array}$ & PFO0II8 & $\begin{array}{l}\text { TCP-I/cpn60 } \\
\text { chaperonin }\end{array}$ & 23 & 34.8 & 17.9 \\
\hline PF00928 & $\begin{array}{l}\text { Adaptor } \\
\text { complexes }\end{array}$ & PFOI2I7 & Clathrin adaptor & 20 & - & 21.7 \\
\hline PF00076 & rrml & PFOI974 & $\begin{array}{l}\text { tRNA intron } \\
\text { endonuclease }\end{array}$ & 18 & - & 3.0 \\
\hline PF00076 & $\mathrm{rrml}$ & PF06220 & zf-UI & 18 & - & 6.2 \\
\hline PFOI 602 & $\begin{array}{c}\text { Adaptin } \mathrm{N} \\
\text { terminal region }\end{array}$ & PFOI2I7 & $\begin{array}{l}\text { Clathrin adaptor } \\
\text { complex }\end{array}$ & 16 & - & 9.2 \\
\hline PF00I 25 & histone & PF00956 & $\begin{array}{c}\text { Nucleosome } \\
\text { assembly protein }\end{array}$ & 16 & - & 14.5 \\
\hline
\end{tabular}

expectation score of each domain interaction are randomly distributed, allowing us to (i) treat the static layout of links and their weights as separate entities and (ii) conclude that protein interactions are indeed governed by a single protein domain interaction [15].

The presence of highly reliable domain interactions offers potential new ways for the prediction and evaluation of protein interactions. In particular, we observe a correlation between an elevated confidence level of a protein interaction in yeast and fly and an increase in the reliability of the underlying domain interactions. As an application, we propose a novel method for the inference of potential protein interactions. While this method can be applied to the prediction of protein interactions in any organism for which PFAM annotation of the organisms proteome is available, we chose the human malaria parasite $P$. falciparum. Screening through all pairs of proteins that provide at least one high scoring domain interaction, we sample potential candidates. Here, we stress that the determination of a high scoring domain interaction has been used as a preselection step of potential protein interaction candidates. In order to evaluate each interaction we resort to interaction specific parameters that are independent from the underlying domain interactions. We find interactions between proteins, that not only show an elevated degree of functional similarity and evolutionary conservation, but also validate our assumption that high scoring domain interactions indeed give rise to reliable interactions. Predominately, we find an enrichment of protein interactions caused by domain interactions that represent functions in the ribosome, proteasome and spliceosome. As reported in protein complexes in other eukaryotes, these functions emphasize a considerable amount of self interactions, we also find in our predictions.

Comparing with existing experimental data sets, we only find a minimal overlap, caused by the fact that many proteins of $P$. falciparum currently are not annotated with PFAM domains. On the other hand, experimental determination of protein interactions in P. falciparum is in its starting phase covering about a quarter of known proteins. As such, our predictions can help focus experimental studies on specific interactions unique to this pathogen. 

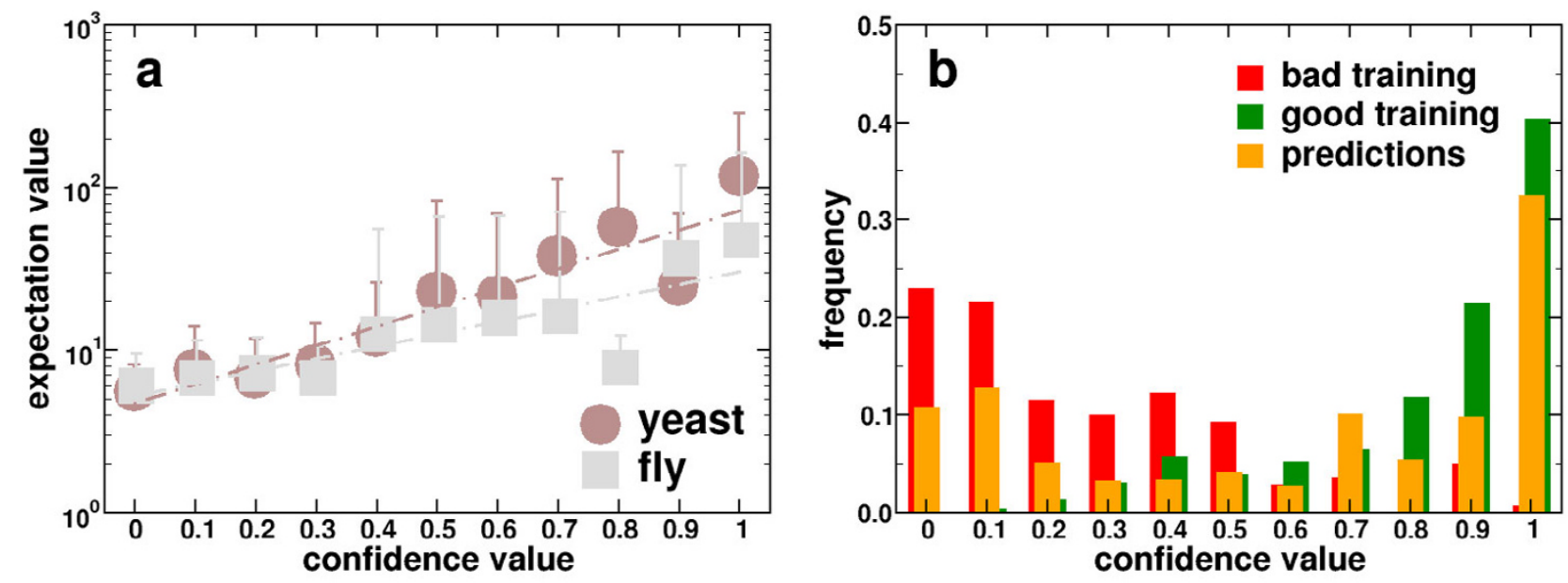

Figure 4

Expectation score vs. confidence in protein interactions of yeast and fly and statistics of training sets and predictions in Plasmodium. (a). Assigning each protein interaction the domain interaction with highest expectation value, we observe that the confidence in the underlying protein interaction correlates with the expectation value of the highest scoring interacting domain pair. In particular, the dependence of the mean domain expectation value $E$ in each bin of confidence values of yeast protein interactions follows a statistically significant exponential distribution $\left(E \sim \mathrm{e}^{(2.73 \times c v), P e a r s o n ' s ~} r=0.28, P<10^{-5}\right.$, Spearman's $\left.\rho=0.3 \mathrm{I}, P<10^{-5}\right)$. In principle, we obtain similar results for fly protein interactions (inset) $\left(E \sim \mathrm{e}^{(1.75 \times c v)}, r=0.19\right.$, $\left.P<10^{-5} ; \rho=0.16, P<10^{-5}\right)$, allowing us to conclude that modeling a protein interaction by the highest scoring domain interaction is a suffcient approximation for the determination of the presence and quality of the underlying protein interaction. Error bars correspond to standard deviations in each bin. (b) In order to evaluate predicted interactions in P. falciparum, we utilized a logistic regression model that we trained by carefully selected sets of true positive and negative interactions. Binning confidence values, we show the frequencies of the predicted protein interactions, the positive (good) and negative (bad) training sets.

\section{Methods}

Domain-domain interactions

As a source of high quality interaction data of protein domains we utilized the results of a recent study by Riley et al. [7]. In this statistical approach called domain pair exclusion analysis (DPEA), a likelihood ratio test is applied to assess the contribution of each potential PFAMA and PFAM-B domain [8] interaction to the likelihood of a set of observed protein interactions. DPEA consists of three steps: (i) Utilizing protein interaction data from DIP [9], the frequency $S_{i j}$ of an interaction between $i$ and $j$ in relation to their abundance in the data is computed. (ii) Using $S_{i j}$ as an initial guess, an expectation maximization algorithm is applied to obtain a maximum likelihood estimate of $\Theta_{i j}$ which stands for the probability of domain interaction $i j$ among all the possible domain interactions which are suggested by the domain architectures of the interacting protein pairs where domain $i$ and $j$ co-occur. In a third step, all possible interactions of domains $i$ and $j$ are excluded from the mixture of competing hypotheses for the presence of corresponding protein interactions, EM is rerun, and the change in likelihood is expressed as a log odds score, $E_{i j}$, reflecting the confidence that domains $i$ and $j$ indeed interact. As such, a high value of $E_{i j}$ indicates that there is extensive evidence in protein interaction data that domains $i$ and $j$ interact while a low $E_{i j}$ suggests that other potential domain interactions are roughly as good at explaining the observed protein interactions [7]. As a proof of concept, domain pairs inferred to interact with high $E$ are significantly enriched among domain pairs known to interact in the Protein Data Bank (PDB). The domain interaction network thus obtained comprises 1 , 566 domains which are embedded in 2, 767 interactions that score $E_{i j} \geq 3$.

\section{Protein interactions}

We utilized a large scale compilation of yeast protein interactions. In particular, this data set combines 47, 783 experimentally obtained protein interactions among 4, 175 proteins in S. cerevisiae [16] obtained from sources as diverse as mRNA expression studies and yeast2hybrid screens. Each interaction was characterized by a confidence score obtained by the application of a logistic regression model. Analogously, the quality of experimentally protein interactions in D. melanogaster was assessed, allowing for 6, 222 proteins and 16, 914 links [17]. As for 

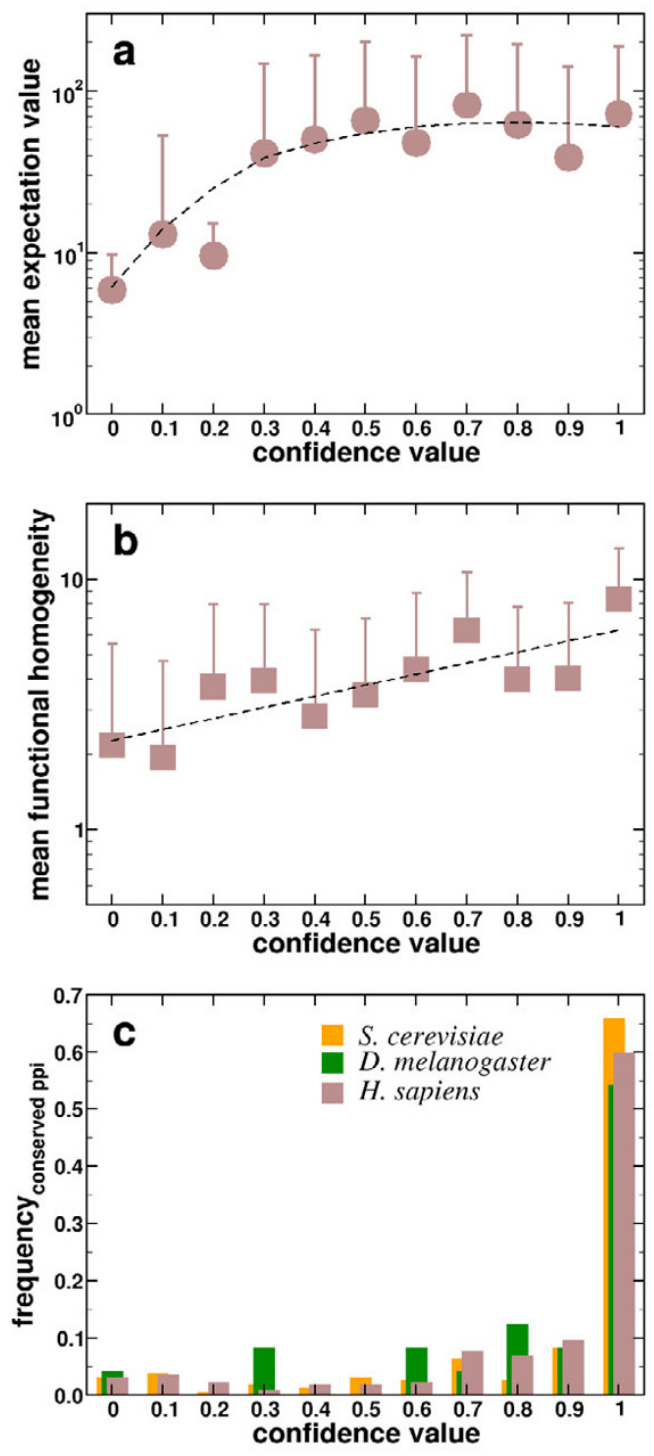

\section{Figure 5}

Statistics of predicted interactions in Plasmodium

(a). In each bin, we calculated the mean expectation value of domain interactions that govern the underlying protein interactions. In particular we obtain a significant correlation $(r=$ $\left.0.22, P<10^{-5}, \rho=0.34, P<10^{-5}\right)$. Error bars correspond to standard deviations in each bin. (b) In the same way, we calculated the mean functional homogeneity, a measure that reflects the probability that GO terms of interacting proteins are similar. In particular, we find a statistically significant correlation $\left(r=0.45, P<10^{-5}, \rho=0.52, P<10^{-5}\right)$. (c) Determining the frequency of interactions which are fully conserved in the organisms $S$. cerevisiae, $D$. melanogaster and $H$. sapiens we find a strong tendency toward evolutionary conservation of predicted interactions with elevated level of confidence. direct experimental observations of protein interactions in $P$. falciparum, we utilized a set of 2, 475 interactions among 1, 304 proteins that have been obtained by the modification of a yeast2hybrid method [27]. Additionally, we utilized a large-scale compilation of human interactions totaling 89, 572 interactions among 9, 018 proteins $[23,24]$.

\section{Protein domain data}

The advent of fully sequenced genomes of various organisms has facilitated the investigation of proteomes. The Integr8 database has been set up to provide comprehensive statistical and comparative analyzes of complete proteomes of fully sequenced organisms. The initial version of the application contained data for genomes and proteomes of 182 sequenced organisms (including 19 archae, 150 bacteria and 13 eukaryotes) and proteome analyzes derived through the integration of UniProt [31], InterPro [32], CluSTr [33], GO/GOA [34], EMSD, Genome Reviews and IPI [35]. In particular, we utilized IPI (International Protein Index) files to elucidate the domain architecture of the corresponding proteins. For our analysis, we focused on domain data retrieved from the PFAM database, a reliable collection of multiple sequence alignments of protein families and profile hidden Markov models [36].

\section{Orthologous protein data}

The InParanoid database [25] provides putative orthologous sequence information for the complete proteomes of organism pairs S. cerevisiae, D. melanogaster, H. sapiens and $P$. falciparum. The algorithm for detecting orthologous relationships is based on pairwise similarity scores which are by default calculated with the BLASTP program. InParanoid detects mutual best hits between sequences from two different species. These are two main orthologs that form an orthologous group. Other sequences are added to this group if they are closely related to one of the main orthologs. These members of the orthologous group are called in-paralogs. A confidence value provided by a standard bootstrap procedure for each in-paralog shows how closely related it is to the main ortholog. In our study, we only selected the main sequence pairs of each orthologous group allowing us to obtain 2, 319 yeast proteins, 1, 351 in D. melanogaster and 1, 525 in H. sapiens with putative orthologs in $P$. falciparum.

\section{Co-expression data}

Genes with similar expression profiles are likely encoding interacting proteins. For $P$. falciparum, we utilized gene expression data, compiling 5, 156 genes over 48 time points as of Winzeler et al., $[19,21]$ and of Bozdech et al. collecting 4, 318 genes over 48 time points [37]. As a gene similarity metric we calculated Pearson's correlation coef- 
fcient for every protein interaction over $m$ time points defined as

$$
r_{p}=\frac{\frac{1}{m} \sum_{i=1}^{m} x_{i} y_{i}-\langle x\rangle\langle y\rangle}{\sigma_{i} \sigma_{j}}
$$

where $\langle x$ Ranglebracket; and $\langle y$ Ranglebracket; are the sample means of expression values $x_{i}$ and $x_{j}$, and $\sigma_{i}$ and $\sigma_{j}$ are their standard deviations.

\section{Logistic regression}

In order to get an estimate of an interactions reliability, we employed a logistic regression model. According to the logistic regression, the probability of a true interaction $T_{v w}$ given the two input variables, hypergeometric clustering coeffcient $x_{1}=C_{v w}$ and co-expression correlation coeffcient $x_{2}=r_{P}, X=\left(x_{1}, x_{2}\right)$

$$
\operatorname{Pr}\left(T_{v w} \mid X\right)=\frac{\exp \left(\beta_{0}+\beta_{1} x_{1}+\beta_{2} x_{2}\right)}{1+\exp \left(\beta_{0}+\beta_{1} x_{1}+\beta_{2} x_{2}\right)}
$$

where $\beta_{n}$ are the parameters of the distribution. Given training data we optimized the distribution parameters by maximizing the likelihood of the data. Here, we applied the corresponding routines as of the Biopython package [38]. As a training set for true positives we choose 213 high scoring protein-interactions in yeast [16] that are fully conserved in Plasmodium. In the same way, we selected 173 low scoring interactions as true negative training set. Applying a leave-one-out analysis to determine the prediction accuracy, our model is recalculated from the training data after removing the interaction to be predicted (leave-one-out), allowing us to obtain the correct result in $95 \%$ of cases.

\section{Hypergeometric clustering coeffcient}

Recently, a network topology based approach uncovered a remarkable correlation between enhanced quality of protein interactions and the degree of clustering of their immediate network neighborhood [22]. Considering a network with $N$ nodes, we define the hypergeometric clustering coeffcient as

$$
C_{v w}=-\log \sum_{i=|N(v) \cap N(w)|}^{\min (|N(v)|, \mid N(w)) \mid\left(\begin{array}{c}
|N(v)| \\
i
\end{array}\right)\left(\begin{array}{c}
N-|N(v)| \\
|N(w)|-i
\end{array}\right)}
$$

where $N(x)$ represents the neighborhood of a vertex $x$. Given fixed neighborhood sizes $N(v)$ and $N(w)$ of nodes $v$ and $w$, the hypergeometric clustering coeffcient increases with elevated overlap between the nodes neighborhoods. Provided that the neighborhoods are independent, the summation can be interpreted as a $p$ value, reflecting the probability of obtaining a number of mutual neighbors between nodes $v$ and $w$ at or above the observed number by chance.

\section{GO annotation data and functional homogeneity}

Similarly to the hypergeometric clustering coeffcient, we define the functional homogeneity of a domain pair $i j$

$$
f h_{i j}=-\log \sum_{i=|G O(v) \cap G O(w)|}^{\min (|G O(v)|, G O(w) \mid)} \frac{\left(\begin{array}{c}
|G O(v)| \\
i
\end{array}\right)\left(\begin{array}{c}
T-|G O(v)| \\
|G O(w)|-i
\end{array}\right)}{\left(\begin{array}{c}
T \\
|G O(w)|
\end{array}\right)}
$$

where $G O(i)$ is the set of GO Terms of protein $i$, and $T$ is the total number of different GO terms [18]. In analogy, the summation can be interpreted as a $p$ value, reflecting the probability that a protein pair shares a certain number of GO terms at or above the observed number by chance.

\section{Additional material}

\section{Additional File 1}

Predicted protein interactions in P. falciparum. This file contains the full set of predicted protein interactions in $\mathrm{P}$. falciparum. Each column is tab-delineated, presenting the interacting proteins (column 1\&2), the underlying domain interactions (columns $3 \& 4$ ), their expectation score (column 5), the interactions confidence score (column 6 ) and the proteins functional annotation (columns $7 \& 8$ )

Click here for file

[http://www.biomedcentral.com/content/supplementary/1471-

2164-7-122-S1.TXT]

\section{Acknowledgements}

This project was entirely funded by the Northwestern Institute on Complexity (NICO).

\section{References}

I. Barabas A, Oltvai Z: Network Biology: Understanding the Cell's Functional Organization. Nat Rev Genet 2004: I0I-I I3.

2. Wuchty S: Topology and Evolution in Yeast Interaction Networks. Genome Res 2004, 14:1310-1314.

3. Han J, Bertin N, Hao T, Goldberg DS, Berriz G, Zhang L, Dupuy D, Walhout A, Cusick M, Roth F, Vidal M: Evidence for dynamically organized modularity in the yeast protein-protein interaction network. Nature 2004, 430:88-93.

4. Guimera R, Amaral L: Functional cartography of complex metabolic networks. Nature 2005, 433:895-900.

5. Wuchty S, Oltvai Z, Barabási AL: Evolutionary conservation of motif constituents within the yeast protein interaction network. Nature Genetics 2003, 35:176-179.

6. Barrat A, Barthélemy M, Pastor-Satorras R, Vespignani A: The architecture of complex weighted networks. Proc Natl Acad Sci USA 2004, I0I(II):3747-3752.

7. Riley R, C Lee CS, Eisenberg D: Inferring protein domain interactions from databases of interacting proteins. Genome Biol 2005, 6(10):R89.

8. Bateman A, Coin L, Durbin R, Finn R, Hollich V, Griffths-Jones S, Khanna A, Marshall M, Moxon S, Sonnhammer E, Studholme D, Yeats C, Eddy S: The Pfam protein families database. Nucl Acids Res 2004, 32:DI38-DI4I. 
9. Xenarios I, Salwinski L, Duan X, Higney P, Kim SM, Eisenberg D: DIP, the Database of Interacting Proteins: a research tool for studying cellular networks of protein interactions. Nucl Acids Res 2002, 30:303-305.

10. Park J, Lappe M, Teichmann S: Mapping Protein Family Interactions: Intramolecular and Intermolecular Protein Family Interaction Repertoires in the PDB and Yeast. J Mol Biol 200I, 307:929-938.

II. Albert R, Barabási AL: Statistical mechanics of complex networks. Rev Mod Phys 2002, 74:47.

12. Watts $D$, Strogatz $S$ : Collective dynamics of 'small-world' networks. Nature 1998, 393:440-442.

13. Newman M: Assortative mixing in networks. Phys Rev Lett 2002 89:20870I.

14. Barthelemy M, Gondran B, Guichard E: Spatial structure of the internet traffc. Physica A 2003, 31 9:633-642.

15. Aloy P, Böttcher B, Ceulemans H, Leutwein C, Mellwig C, Fischer S, Gavin AC, Bork P, Superti-Furga G, Serrano L, Russell R: StructureBased Assembly of Protein Complexes in Yeast. Science 2004, 303:2026-2029.

16. Bader JS, Chaudhuri JRD, Chant J: Gaining confidence in highthroughput protein interaction networks. Nat Biotechnol 2004 22:78-85.

17. Giot L, Bader J, Brouwer C, Chaudhuri A, Kuang B, Li Y, Hao Y, Oo C, Godwin B, Vitols E, Vijayadamodar G, Pochart P, Machineni $H$ Welsh M, Kong Y, Zerhusen B, Malcolm R, Varrone Z, Collis A, Minto M, Burgess S, McDaniel L, Stimpson E, Spriggs F, Williams J, Neurath K, loime N, Agee M, Voss E, Furtak K, Renzulli R, Aanensen N, Carrolla S, Bickelhaupt E, Lazovatsky Y, DaSilva A, Zhong J, Stanyon C, Finley R Jr, White K, Braverman M, Jarvie T, Gold S, Leach M, Knight J, Shimkets R, McKenna M, Chant J, Rothberg J: A Protein Interaction Map of Drosophila melanogaster. Science 2003, 302: 1727-1736.

18. Consortium GO: The Gene Ontology (GO) database and informatics resource. Nucl Acids Res 2004, 32:D258-D261.

19. Le Roch K, Zhou Y, Blair P, Grainger M, Moch J, Haynes J, De la Vega P. Holder A, Batalov S, Carucci D, Winzeler E: Discovery of Gene Function by Expression Profiling of the Malaria Parasite Life Cycle. Science 2003, 301:1503-1508.

20. Johnson KLJ, Florens L, Zhou Y, Santrosyan A, Grainger M, Yan S, Williamson K, Holder A, Carucci D, Yates III J, Winzeler E: Global analysis of transcript and protein levels across the Plasmodium falciparum life cycle. Genome Res 2004, 14:2308-23 I8.

21. Winzeler E: Applied systems biology and malaria. Nat Rev Microbiol 2006, 4:|45-I5|.

22. Goldberg D, Roth F: Assessing experimentally derived interactions in a small world. Proc Natl Acad Sci USA 2003, 100:4372-4376.

23. Lehner B, Fraser A: A first-draft human protein-interaction map. Genome Biol 2004, 5(9):R63.

24. Ramani A, Bunescu R, Mooney R, Marcotte E: Consolidating the set of known human protein-protein interactions in preparation for large-scale mapping of the human interactome. Genome Biol 2005, 6(5):R40.

25. Remm M, Storm C, Sonnhammer E: Automatic clustering of orthologs and in-paralogs from pairwise species comparisons. J Mol Biol 200I, 3 I 4: 104I-1052.

26. Ge H, Liu Z, Church G, Vidal M: Correlation between transcriptome and interactome mapping data from Saccharomyces cerevisiae. Nature Genetics 200I, 29:482-486.

27. LaCount D, Vignali M, Chettier R, Phansalkar A, Bell R, Hesselberth J, Schoenfeld L, I Ota SS, Kurschner C, Fields S, Hughes R: A protein interaction network of the malaria parasite Plasmodium falciparum. Nature 2005, 438: 103-107.

28. Bochtler M, Ditzel L, Groll M, Hartmann C, Huber R: The Proteasome. Annu Rev Biophys Biomol Struct 1999, 28:295-317.

29. Matadeen R, Patwardhan A, Gowen B, Orlova E, Pape T, Cuf M, Mueller $F$, Brimacombe $R$, van Heel M: The Escherichia coli large ribosomal subunit at 7.5A resolution. Structure Fold Des 1999, 7:1575-1583

30. Mura C, Cascio D, Sawaya M, Eisenberg D: The crystal structure of a heptameric archaeal SM protein: implications for the eukaryotic snRNP core. Proc Natl Acad Sci USA 2001, 98:5532-5537.

31. Apweiler R, Bairoch A, Wu C, Barker W, Boeckmann B, Ferro S, Gasteiger E, Huang H, Lopez R, Magrane M, Martin M, Natale D,
O'Donovan C, Redaschi N, Yeh L: Uniprot: the universal protein knowledgebase. Nucl Acids Res 2004, 32:DII5-DII9.

32. Mulder N, Apweiler R, Attwood T, Bairoch A, Barrell D, Bateman A, Binns D, Biswas M, Bradley P, Bork P, Bucher P, Copley R, Courcelle E, Das U, Durbin R, Falquet L, Fleischmann W, Griffths-Jones S, Haft D, Harte N, Hulo N, Kahn D, Kanapin A, Krestyaninova M, Lopez R, Letunic I, Lonsdale D, Silventoinen V, Orchard S, Pagni M, Peyruc D, Ponting C, Selengut J, Servant F, Sigrist C, Vaughan R, Zdobnov E: The InterPro Database, 2003 brings increased coverage and new features. Nucl Acids Res 2003, 3 I:3 I5-3I8.

33. Kriventseva E, Fleischmann W, Zdobnov E, Apweiler R: CluSTr: a database of clusters of SWISS-PROT+TrEMBL proteins. Nucl Acids Res 200I, 29:33-36.

34. Consortium GO: The gene ontology (go) database and information resource. Nucl Acids Res 2004, 32:D258-D26I.

35. Kersey P, Duarte J, Williams A, Apweiler R, Karavidopoulou Y, Birney $E$ : The international protein index: An integrated database for proteomics experiments. Proteomics 2004, 4(7): 1985-1988.

36. Bateman A, Coin L, Durbin R, Finn R, Hollich V, Griffths-Jones S, Khanna A, Marshall M, Moxon S, Sonnhammer E, Studholme D, Yeats C, Eddy S: The Pfam protein families database. Nucl Acids Res 2004, 32:DI38-DI4I.

37. Bozdech Z, Llinas M, Pulliam B, Wong E, Zhu J, DeRisi J: The Transcriptome of the Intraerythrocytic Developmental Cycle of Plasmodium falciparum. PLOS Biol 2003, I: I-16.

38. The Biopython package [http://www.biopython.org]
Publish with Bio Med Central and every scientist can read your work free of charge

"BioMed Central will be the most significant development for disseminating the results of biomedical research in our lifetime. "

Sir Paul Nurse, Cancer Research UK

Your research papers will be:

- available free of charge to the entire biomedical community

- peer reviewed and published immediately upon acceptance

- cited in PubMed and archived on PubMed Central

- yours - you keep the copyright

Submit your manuscript here:

http://www.biomedcentral.com/info/publishing_adv.asp
BioMedcentral 